Supporting Information for

\title{
Confronting the Air Instability of Cesium Tin Halide Perovskites by Metal Ion Incorporation
}

Shu Zhou, ${ }^{\mathrm{a}, 1, *}$ Shengcai Zhu, ${ }^{\mathrm{a}, 1}$ Jiuhui Guan, ${ }^{\mathrm{a}, 1}$ Rong Wang, ${ }^{\mathrm{b}}$ Wei Zheng, ${ }^{\mathrm{a}}$ Pingqi Gao, ${ }^{\mathrm{a},{ }^{*}}$ and Xinhui $L u^{\mathrm{c}, *}$

${ }^{\text {a }}$ School of Materials, Shenzhen Campus of Sun Yat-sen University, Shenzhen, 518107, China

${ }^{\mathrm{b}}$ Hangzhou Global Scientific and Technological Innovation Center, Zhejiang University, Hangzhou, 311200, China

${ }^{\mathrm{c}}$ Department of Physics, The Chinese University of Hong Kong, New Territories, Hong Kong

${ }^{1}$ These authors contributed equally to this work.

*E-mails: zhoush67@mail.sysu.edu.cn,gaopq3@mail.sysu.edu.cn,xinhui.lu@cuhk.edu.hk 


\section{Methods}

Simulations: We employ a recently developed stochastic surface walking (SSW) method ${ }^{1,2}$ integrating with the high-dimensional neural networks $(\mathrm{NN})$ potential ${ }^{3}$ and density functional theory (DFT) to clarify the mechanism of phase transition. Details of the algorithm of SSW pathway sampling were described in previous work. ${ }^{4}$ The SSW pathway sampling is fully automated and divided into three stages in simulation as follows, (i) utilize the SSW method to explore all the likely phases nearby the phase; (ii) utilize the variable-cell double-ended surface walking (DESW) method $^{5}$ to establish the pseudo-pathway connecting IS (initial state) to FS (final state) for all IS/FS pairs; (iii) locate exactly the transition state (TS) of the candidate lowest energy pathways by using DESW TS-search method. For the black-to-yellow phase transition in the current work, we have collected more than 10,000 pairs IS/FS which leads to the finding of the lowest energy pathway. For the large number of possible reaction pathways and the large system (20 atoms), SSW method integrated with the HDNN potential is the practical solution for the initial screening of the pathways. All calculations were performed using the plane wave DFT program, Vienna ab initio simulation package (VASP). ${ }^{6}$ The electron-ion interaction is represented by the projector augmented wave (PAW) scheme ${ }^{7}$ and the exchange-correlation function is described by the generalized gradient approximation (GGA) in the Perdew-Burke-Ernzerhof (PBE) parameterization. ${ }^{8}$ During the DFT calculations, the kinetic energy plane wave cutoff is $450 \mathrm{eV}$, Monkhorst-Pack k-point mesh $(3 \times 3 \times 5)$ for 20 -atom supercell while $(1 \times 1 \times 1)$ for 60 -atom supercell for $\mathrm{Sn}^{2+}$ ions diffusion, which mimic the black-phase $\mathrm{CsSnI}_{3}$ to zero dimensional $\mathrm{Cs}_{2} \mathrm{SnI}_{6}$ degradation. For all the structures, both lattice and atomic positions were fully 
optimized until the maximal stress component is below $0.1 \mathrm{GPa}$ and the maximal force component below 0.01 eV/Å.

For the DFT simulations of defect formation energies in black-phase $\gamma-\mathrm{CsSnI}_{3}$, a cell with 80 atoms was adopted. The plane-wave cut-off energies were set as $400 \mathrm{eV}$. The structural optimization and atomic relation were performed with the PBE exchange correlation functional, until the change in total energy was less than $10^{-5} \mathrm{eV}$ per cell. During electronic calculations, the screened hybrid density functional of Heyd, Scuseria and Ernzerhof (HSE06) was employed for accurate description of bandgap energies and defect-level positions. ${ }^{9}$ The reciprocal space of the supercell was sampled by the MonkhorstPack scheme with a $\Gamma$-centered $2 \times 2 \times 2$ special k-points mesh. ${ }^{10}$ The defect formation energies $\mathrm{V}_{\text {Sn }}$ and BiSn were calculated by the mixed k-point scheme. ${ }^{11,12}$

Materials: Tin (II) iodide ( $\mathrm{SnI}_{2}$, Sigma-Aldrich, 99.999\%, metals basis), cesium iodide (CsI, Sigma-Aldrich, 99.999\%, metals basis), bismuth (III) iodide (BiI 3 , Sigma-Aldrich, 98\% metals basis), bismuth (III) bromide $\left(\mathrm{BiBr}_{3}\right.$, Sigma-Aldrich, 98\% metals basis), N,N-dimethylformamide (DMF) (Sigma-Aldrich, anhydrous, 99.8\%), PEDOT:PSS (Heraeus Clevios PH1000), $\mathrm{PC}_{61} \mathrm{BM}$ (American Dye Source), silver (Sigma-Aldrich, 99.99\%).

Fabrication of black $\boldsymbol{\gamma}$-CsSnI 3 films: For preparing undoped $\mathrm{CsSnI}_{3}$ precursor, $\mathrm{CsI}$ and $\mathrm{SnI}_{2}$ were firstly mixed together in 1: 1 molar ratio in a dry nitrogen-filled glovebox. Subsequently, $\mathrm{N}, \mathrm{N}$-dimethylformamide (DMF) was added to make a $0.3 \mathrm{M}$ solution, which was stirred overnight before use. For preparing doped $\mathrm{CsSnI}_{3}$ precursor, $\mathrm{CsI}, \mathrm{SnI}_{2}$ and $\mathrm{BiI}_{3} / \mathrm{BiBr}_{3}$ were firstly mixed together in 1: 1: $(0.01 \sim 0.2)$ molar ratio. To deposit $\mathrm{CsSnI}_{3}$ films, two drops of 
solution were cast onto a FTO substrate spinning at 4,000 rpm for $60 \mathrm{~s}$. The black phase $\gamma$-CsSnI 3 forms immediately upon solvent evaporation.

Fabrication of devices: FTO glass substrates were cleaned by sonication in deionized water, isopropyl alcohol and acetone, followed by oxygen plasma treatment for $15 \mathrm{~min}$. Immediately after the oxygen plasma treatment, a PEDOT:PSS film was deposited onto the cleaned FTO substrate using a spin speed of $3000 \mathrm{rpm}$. Subsequently, the substrates were transferred into a dry nitrogen-filled glovebox for $\mathrm{CsSnI}_{3}$ film deposition, followed by deposition of a $\mathrm{PC}_{61} \mathrm{BM}$ film from a $15 \mathrm{mg} / \mathrm{ml}$ chlorobenzene solution using a spin speed of $1500 \mathrm{rpm}$. Finally, a 100 $\mathrm{nm}$ Ag electrode was deposited by thermal evaporation.

Characterizations: XRD measurements were performed by Rigaku (Smart Lab). X-rays generated by a $\mathrm{Cu} \mathrm{KR}$ source at the voltage of $40 \mathrm{kV}$ were employed. A scanning step of $0.02^{\circ}$ was used for measuring all the $\mathrm{CsSnI}_{3}$ films. XPS measurements were carried out at BL09A2 U5 beamline at the National Synchrotron Radiation Research Center, Taiwan, with incident photon energy of $750 \mathrm{eV}$. A UV-vis spectrometer (Hitachi U-3501) was used to measure the optical absorption of all $\mathrm{CsSnI}_{3}$ films. SEM images were recorded by using a FEI Quanta 400 field emission microscope operated at an acceleration voltage of $25 \mathrm{kV}$. Hall measurements were carried out with a Lake Shore Hall measurement system (HM 3000) by using the van der Pauw method. Current density-voltage $(\mathrm{J}-\mathrm{V})$ curves were measured under dark condition and AM 1.5G solar illumination at $100 \mathrm{~mW} / \mathrm{cm}^{2}$ by using a Keithley 2612 source meter with a scanning rate of $0.1 \mathrm{~V} / \mathrm{s}$. 

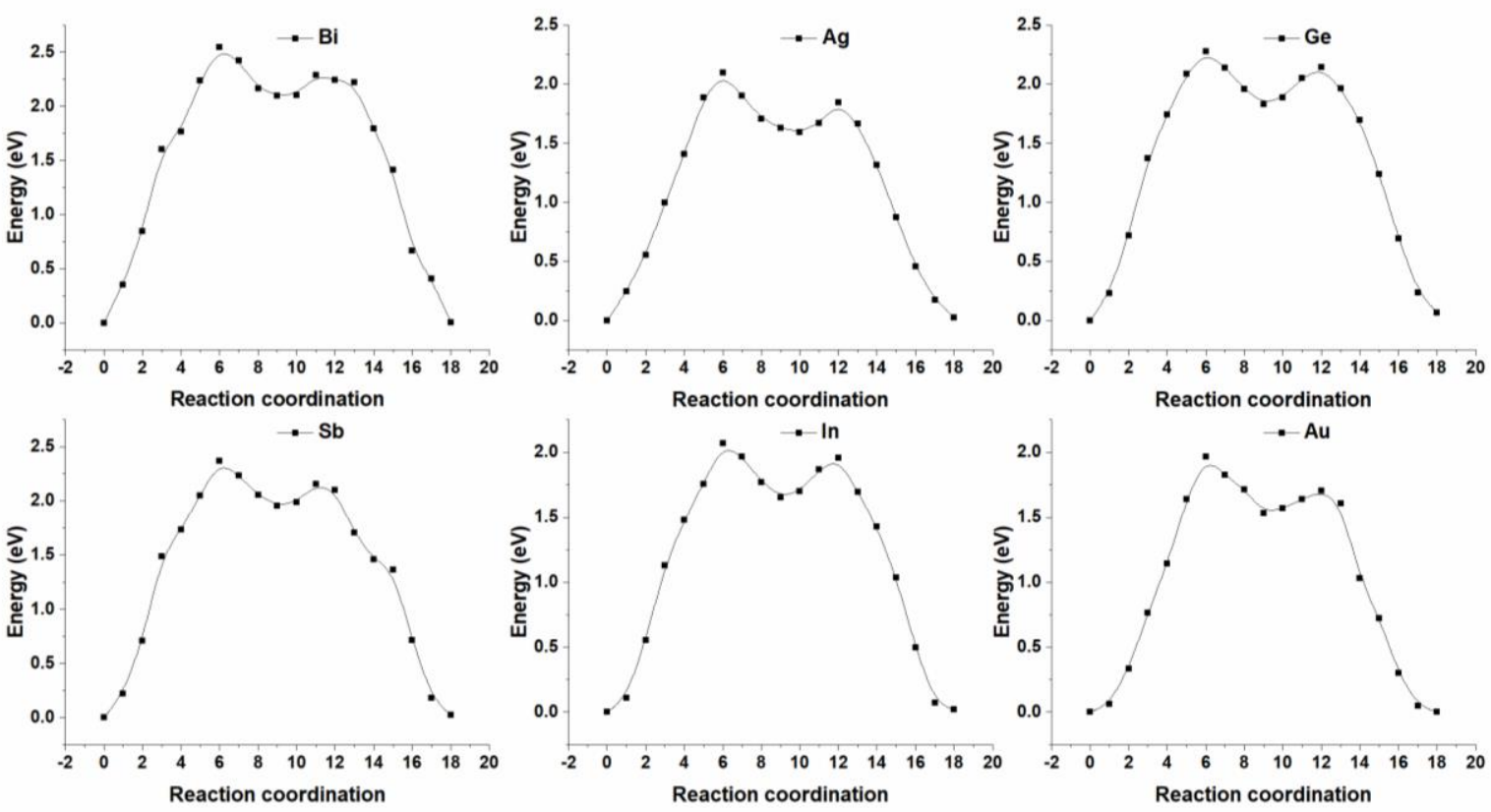

Figure S1. The calculated energy barrier $\left(\mathrm{E}_{2}\right)$ for the structure evolution of black-phase $\mathrm{CsSnI}_{3}$ to zero dimensional $\mathrm{Cs}_{2} \mathrm{SnI}_{6}$ mediated by $\mathrm{Sn}^{2+}$ diffusion. 

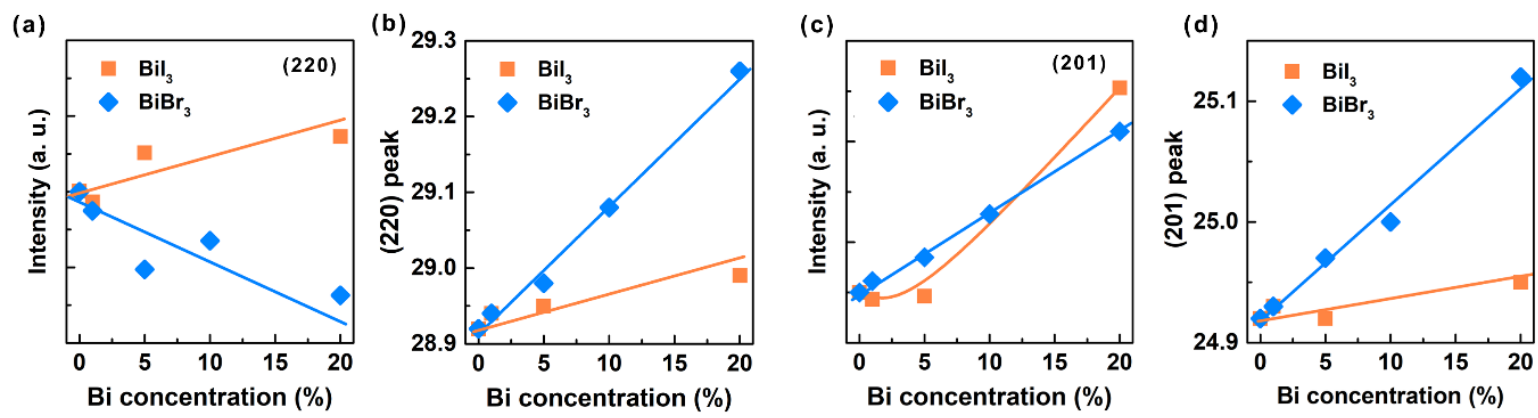

Figure S2. (a-b) Intensity profile and position of the (220) peak with increasing doping concentration. (c-d)

Intensity profile and position of the (201) peak with increasing doping concentration. 

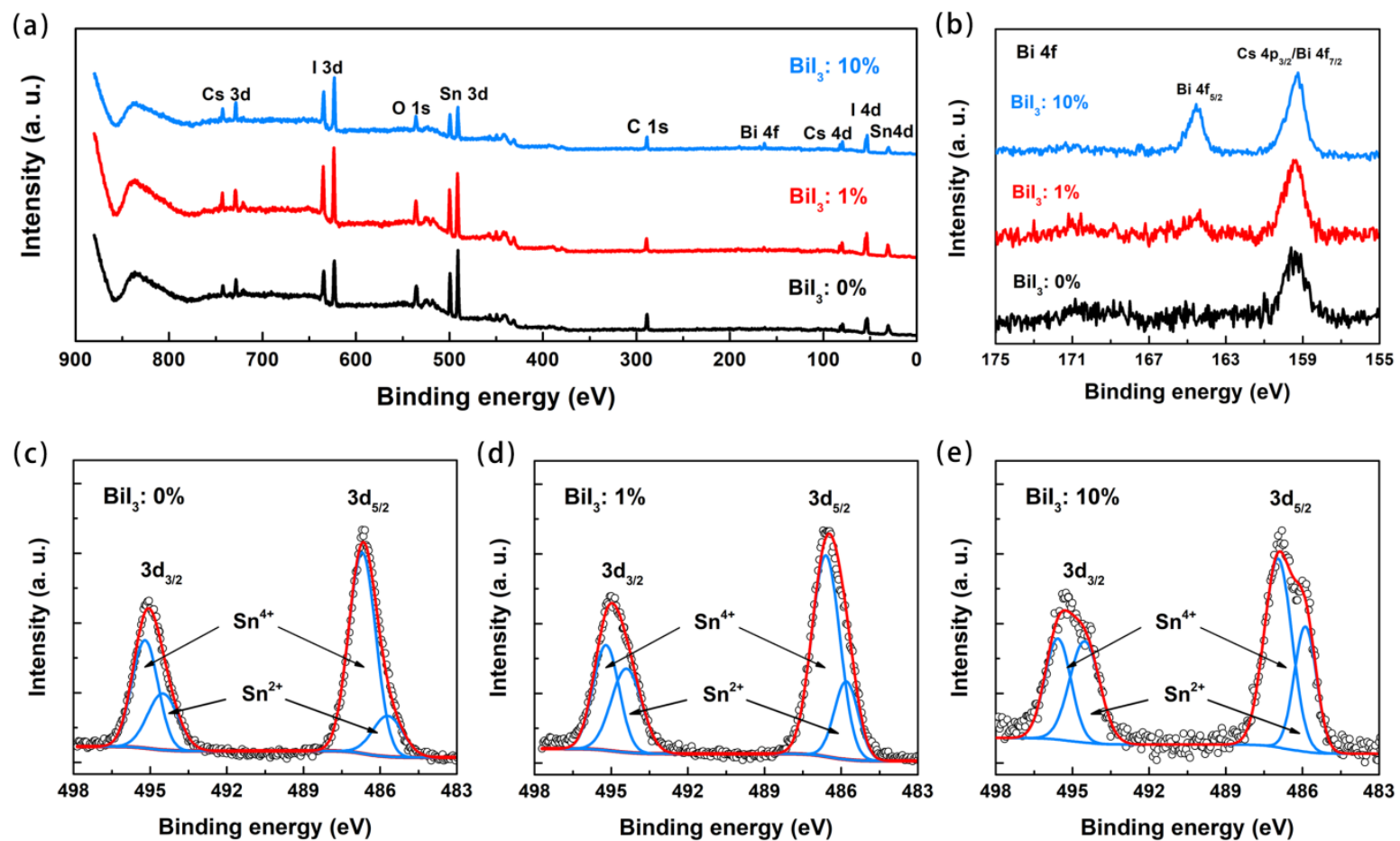

Figure S3. XPS spectra for undoped and $\mathrm{BiI}_{3}$ doped $\gamma$-CsSnI 3 films. (a) Survey, (b) Bi 4f and (c-e) Sn 3d spectrum. All films were shortly exposed to air before transfer into the XPS chamber. 


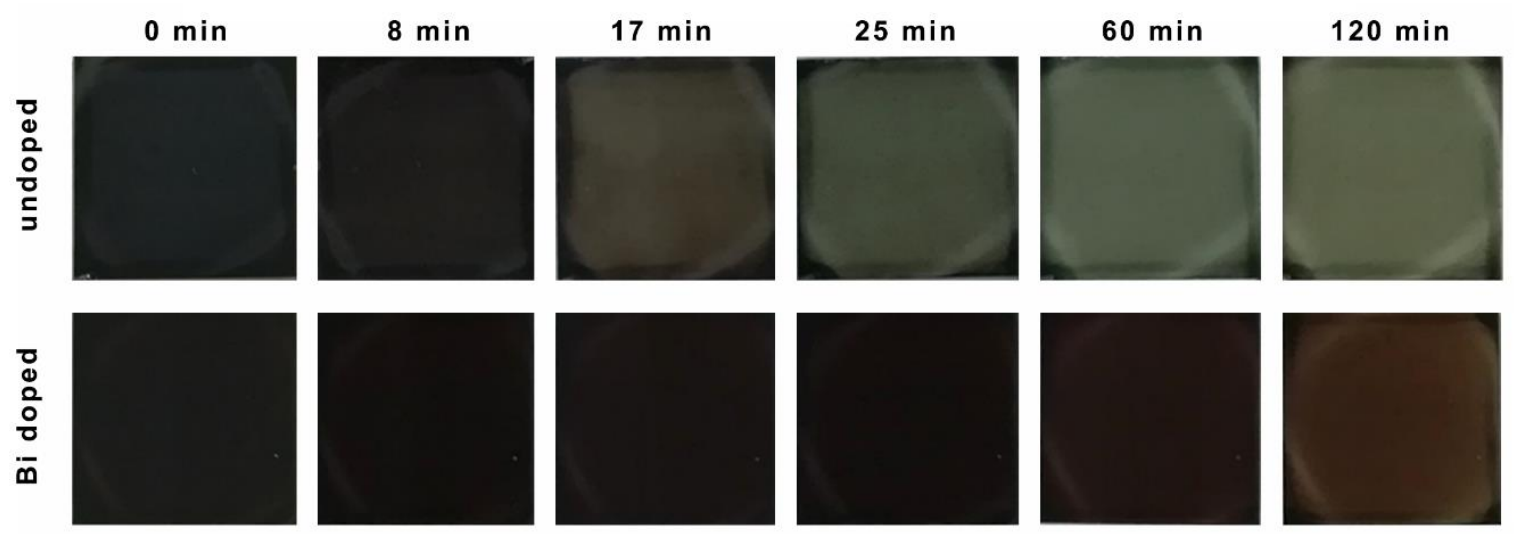

Figure S4. Images of undoped and $\mathrm{BiBr}_{3}$ doped $\mathrm{CsSnI}_{3}$ films in $60-80 \%$ humidity air. 

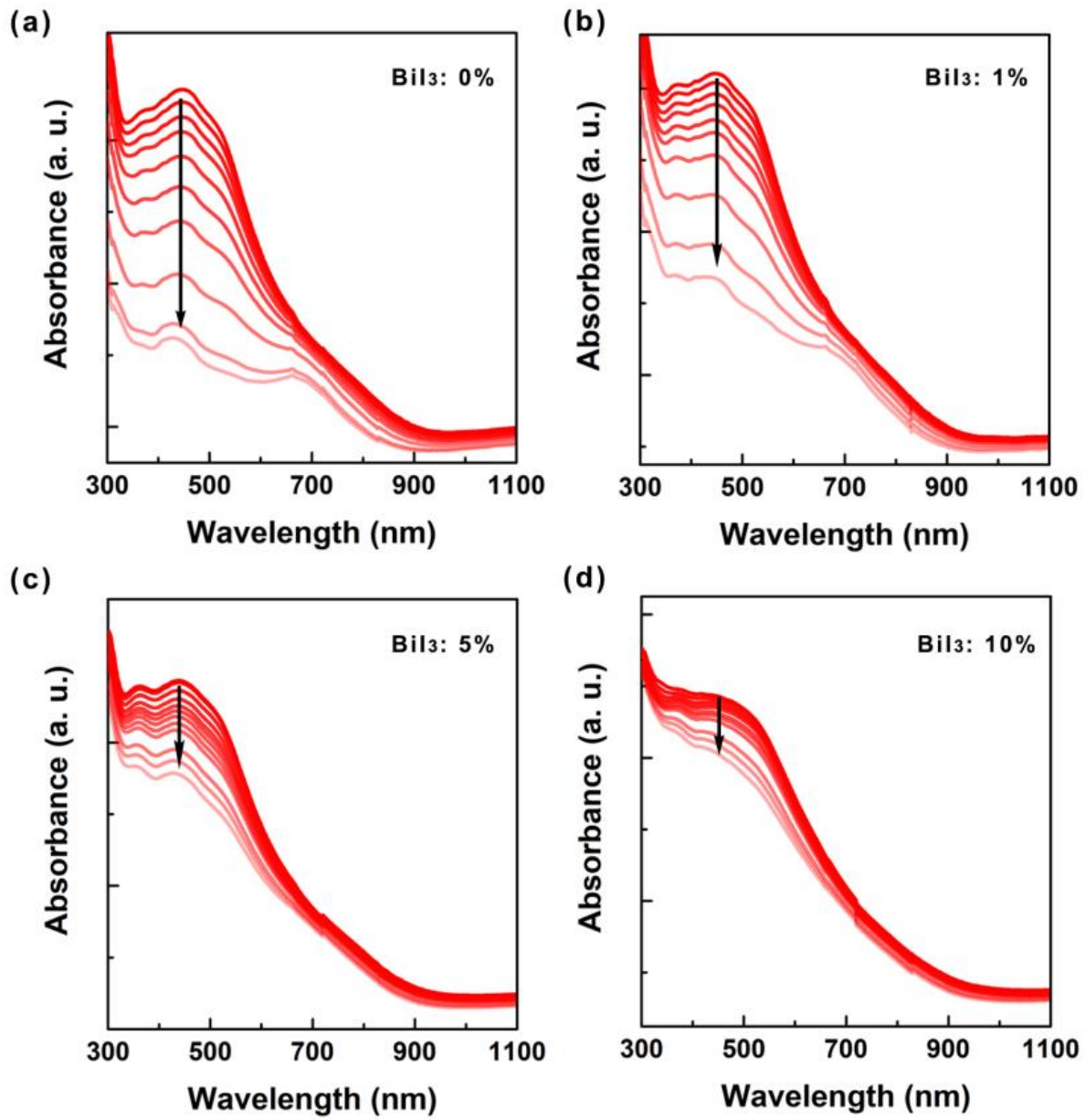

Figure S5. Evolution of the absorption spectrum of $\mathrm{CsSnI}_{3}$ films with different $\mathrm{BiI}_{3}$ concentration in $60-80 \%$ humid air. The black arrows indicate the direction of change with increasing time. All films were supported on fluorine tin oxide (FTO)-coated glass. 

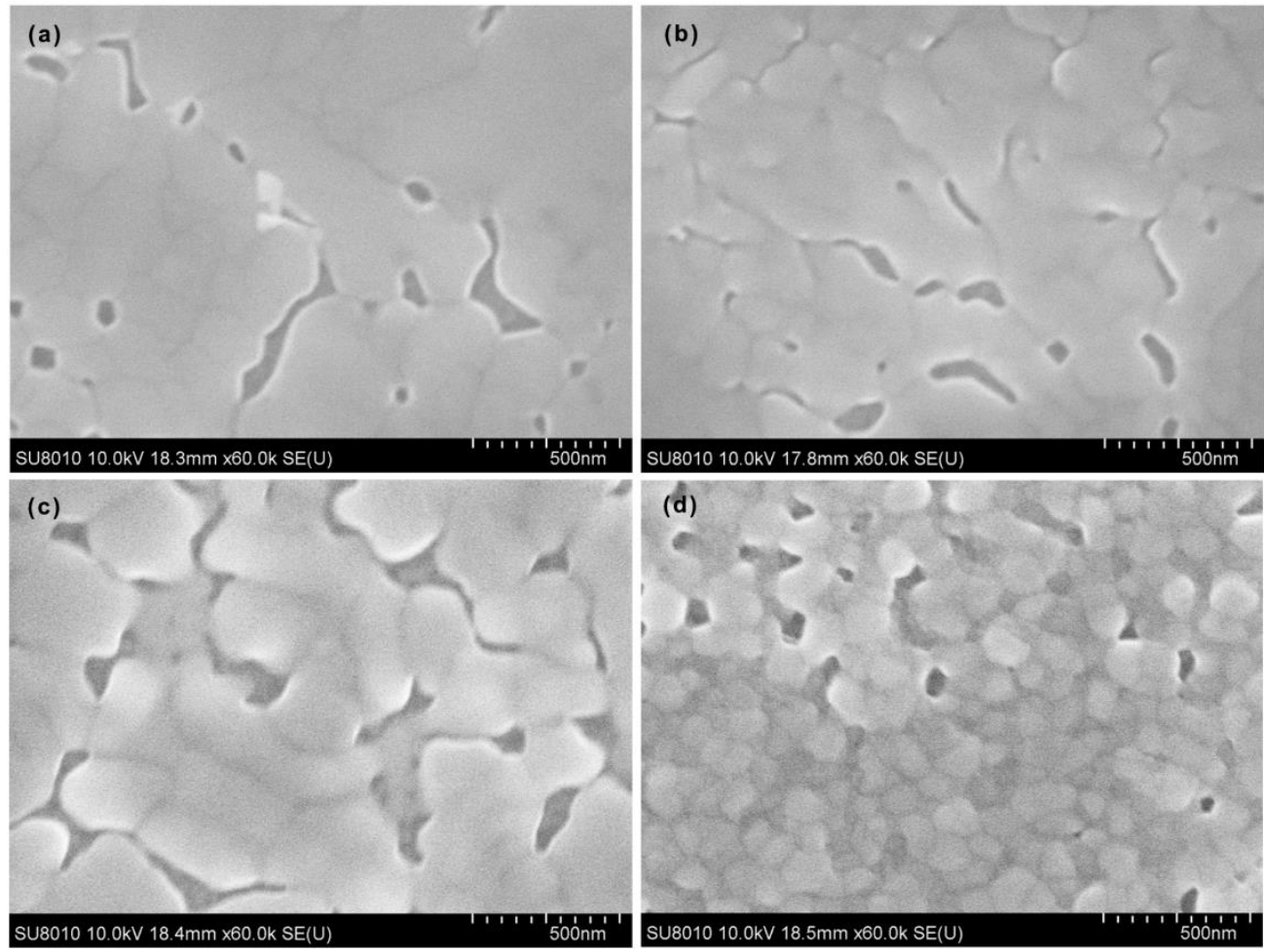

Figure S6. SEM images of undoped and Bi doped $\mathrm{CsSnI}_{3}$ films supported on FTO coated glass. (a) $0 \%$ $\mathrm{BiBr}_{3}$, (b) $1 \% \mathrm{BiBr}_{3}$, (c) $10 \% \mathrm{BiBr}_{3}$ and (d) $20 \% \mathrm{BiBr}_{3}$. 


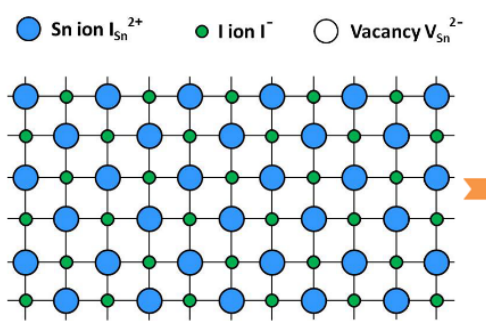

Tin perovskites

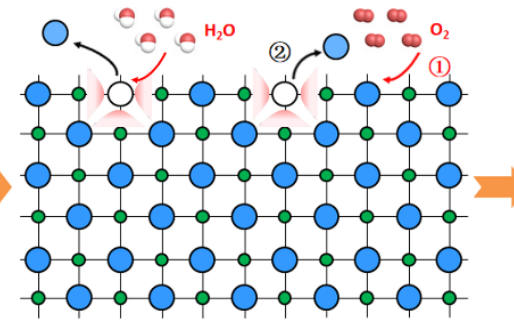

Formation of tin vacancy

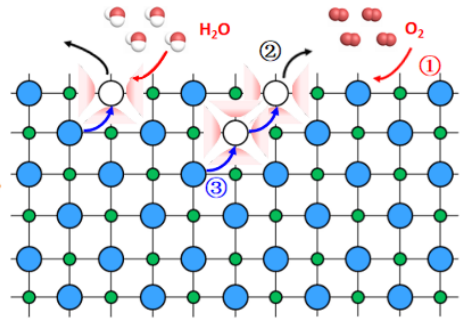

Diffusion of tin vacancy

Figure S7. Schematic illustrating the oxidaion of $\gamma-\mathrm{CsSnI}_{3}$ to $\mathrm{Cs}_{2} \mathrm{SnI}_{6}$ in humidity air. The adsorption of $\mathrm{H}_{2} \mathrm{O}$ leads to desorption of $\mathrm{Sn}^{2+}$ from the surface, creating a $\mathrm{VSn}^{2+}$. The subsequent diffusion of $\mathrm{V}_{\mathrm{Sn}^{2+}}$ toward the subsurface leads to the continuous oxidation of $\mathrm{Sn}^{2+}$ to $\mathrm{Sn}^{4+}$. The local strain induced by $\mathrm{V}_{\mathrm{Sn}^{2+}}$ that promotes the degradation is highlighted.

As depicted in Figure S7, the adsorption of $\mathrm{H}_{2} \mathrm{O}$ and $\mathrm{O}_{2}$ (path (1)) leads to desorption of $\mathrm{Sn}^{2+}$ (path (2)) from the perovskite surface. $\mathrm{A} \mathrm{V}_{\mathrm{Sn}^{2+}}$ would then be created. The subsequent diffusion of $\mathrm{V}_{\mathrm{Sn}^{2+}}{ }^{2+}$ (path (3) toward the subsurface leads to the continuous oxidation of $\gamma-\mathrm{CsSnI}_{3}$. And more $\mathrm{V}_{\mathrm{Sn}^{2+}}$ will be created, giving rise to a local tensile strain that shifts the diffraction peak to a smaller angle. 
(a)

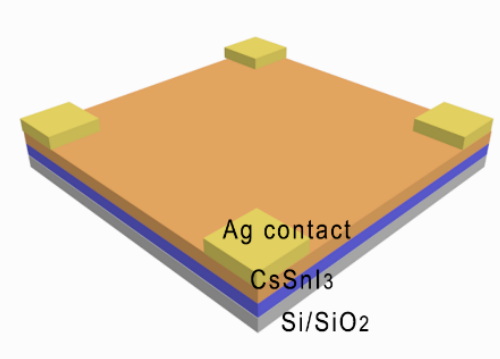

(b)

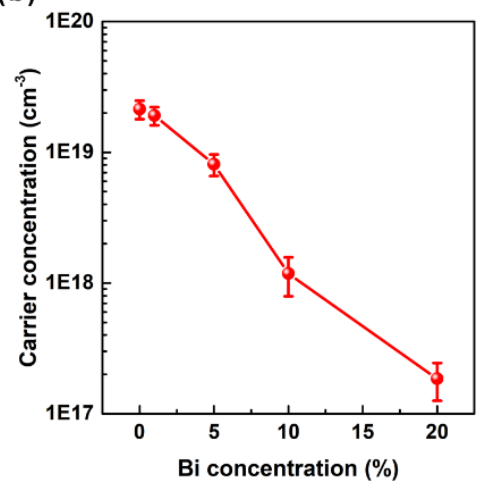

(c)

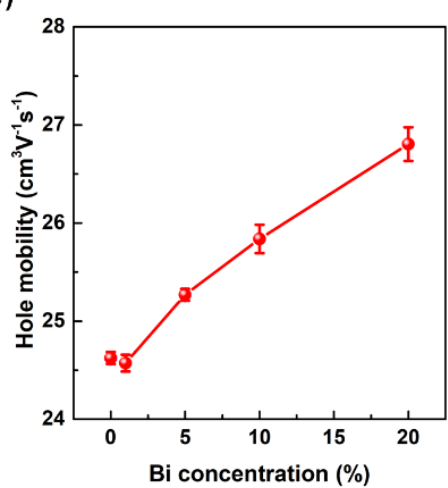

Figure S8. Impact of $\mathrm{BiI}_{3}$ doping on the electrical properties of $\boldsymbol{\gamma}$-CsSnI $\mathbf{C}_{3}$ films. (a) Hall device structure $\left(\mathrm{Ag} / \mathrm{CsSnI}_{3} / \mathrm{Ag}\right)$, (b) carrier concentration and (c) hole mobility for the devices made by using undoped and $\mathrm{Bi}$ doped $\mathrm{CsSnI}_{3}$ films. 


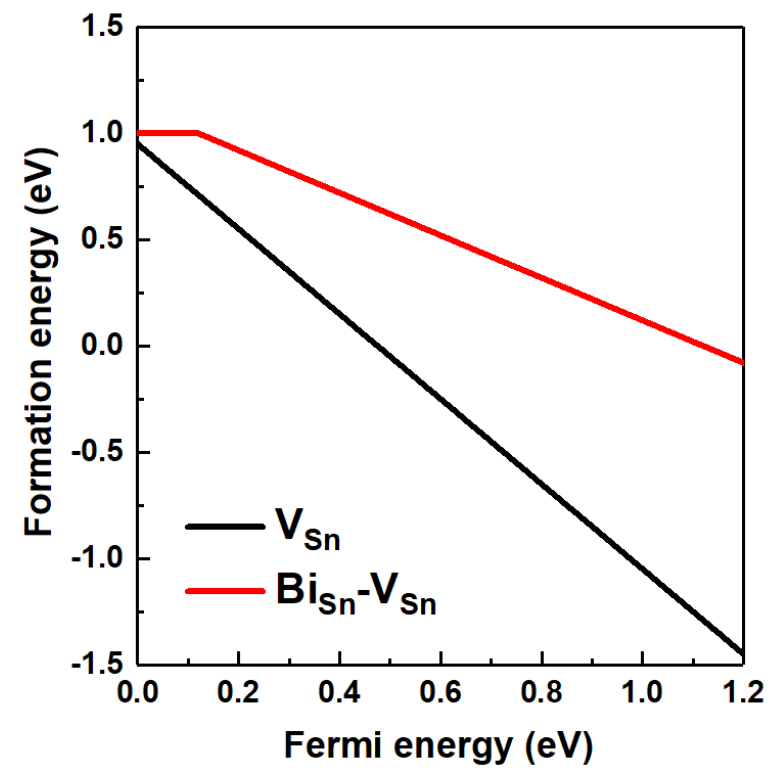

Figure S9. Impact of Bi substituion on the formation energy of tin vacnacy $\left(\mathrm{V}_{\mathrm{Sn}}\right)$ in black-phase $\gamma$-CsSnI ${ }_{3}$.

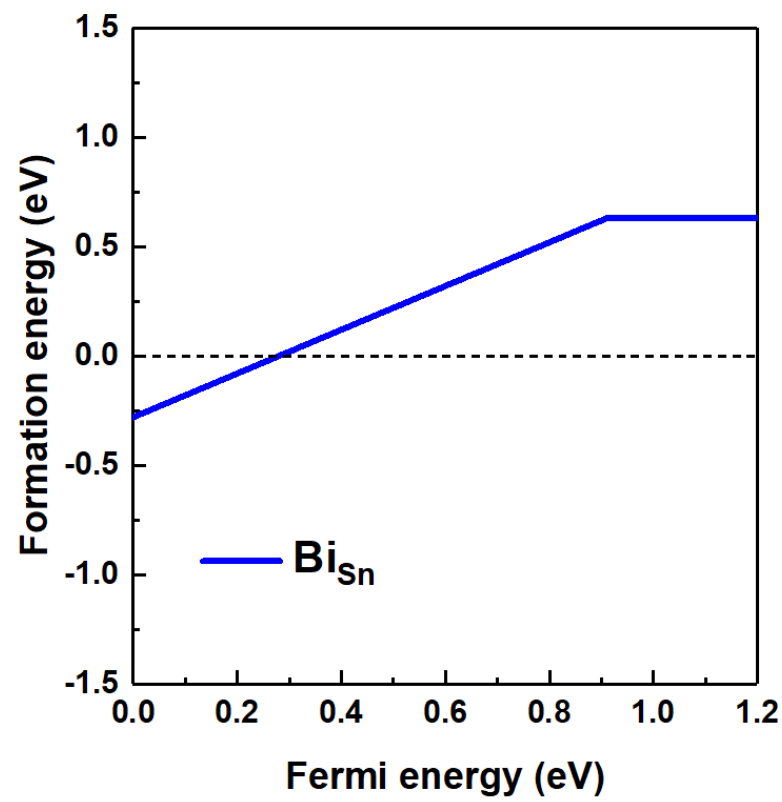

Figure S10. Formation energy for the Bi substitution $(\mathrm{Bi}$ sn $)$ in black-phase $\gamma-\mathrm{CsSnI}_{3}$. 


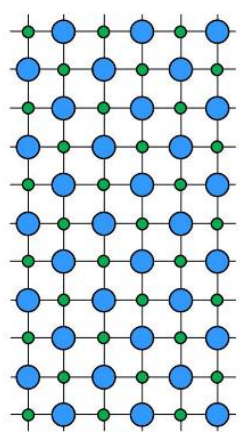

(i)

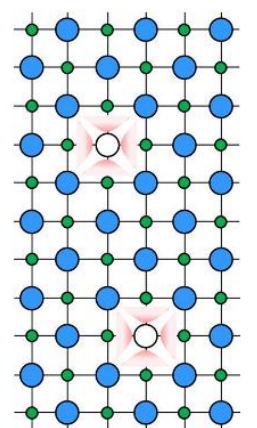

(ii)

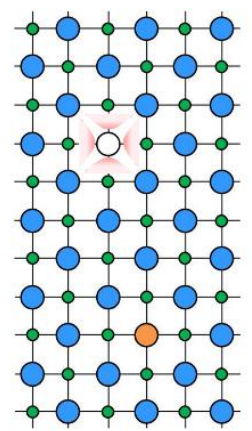

(iii)

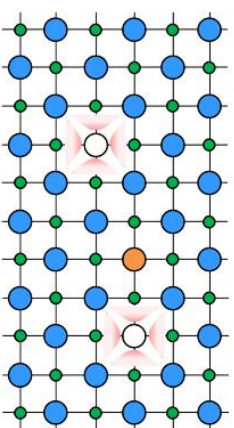

(iv)

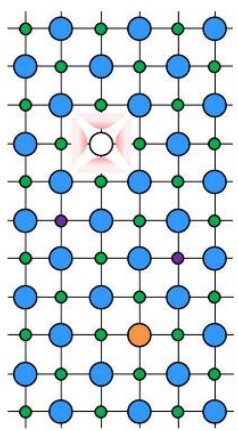

(v)
Sn ion $\mathrm{I}_{\mathrm{s} n}^{2+}$

○Iion $1^{-}$

Vacancy $\mathrm{v}_{\mathrm{sn}_{\mathrm{n}}}^{2-}$

$\mathrm{Bi}$ ion $\mathrm{IBi}^{3+}$

- $\mathrm{Br}$ ion $\mathrm{Br}^{-}$

Figure S11. Schematic showing the possible mechanisms for enhanced stability in Bi doped $\gamma$-CsSnI ${ }_{3}$

films. i. $\mathrm{CsSnI}_{3}$ with a complete chemcal formula, ii. Formation of $\mathrm{V}_{\mathrm{Sn}}{ }^{2+}$ leads to expansion of the lattice, iii. Substitution of a $\mathrm{VSn}_{\mathrm{Sn}^{2+}}$ by a $\mathrm{Bi}^{3+}$, iv. Substitution of a $\mathrm{Sn}^{2+}$ by a $\mathrm{Bi}^{3+}$, v. Substittion of a I- by a $\mathrm{Br}^{-}$. The local strain induced by $\mathrm{V}_{\mathrm{Sn}^{2+}}$ that promotes the degradation is highlighted in ii-v. 
(a)

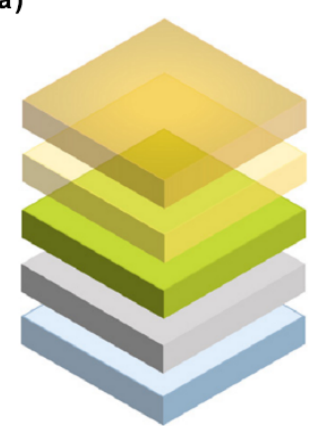

(b)

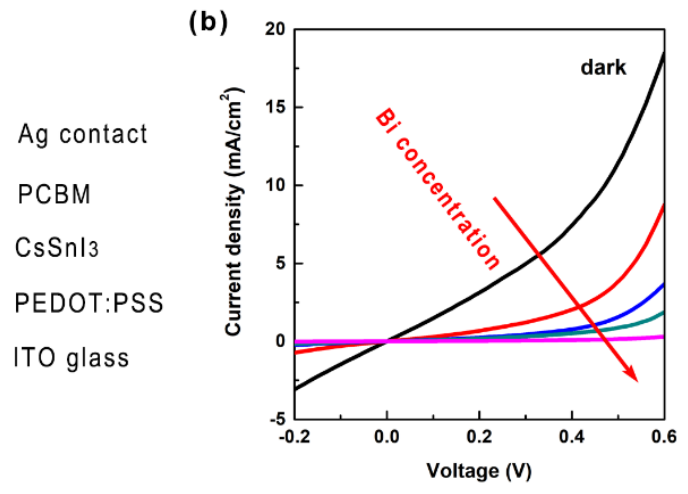

(c)

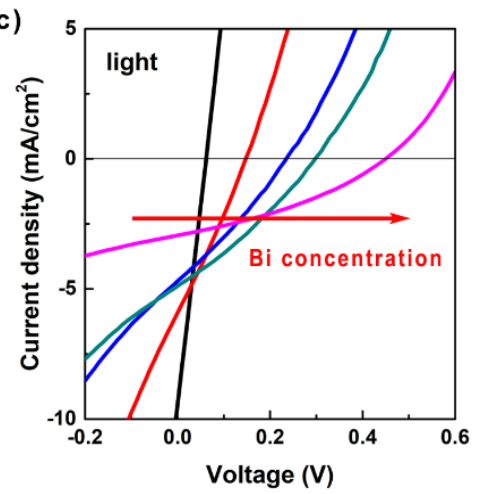

Figure S12. (a) Solar cell device structure (ITO/PEDOT:PSS/CsSnI $3 / \mathrm{PCBM} / \mathrm{Ag}$ ) and current-voltage (I-V) characteristics of the devices made by using undoped and $\mathrm{BiI}_{3}$ doped $\mathrm{CsSnI}_{3}$ films measured under (b) dark and (c) light condition. 


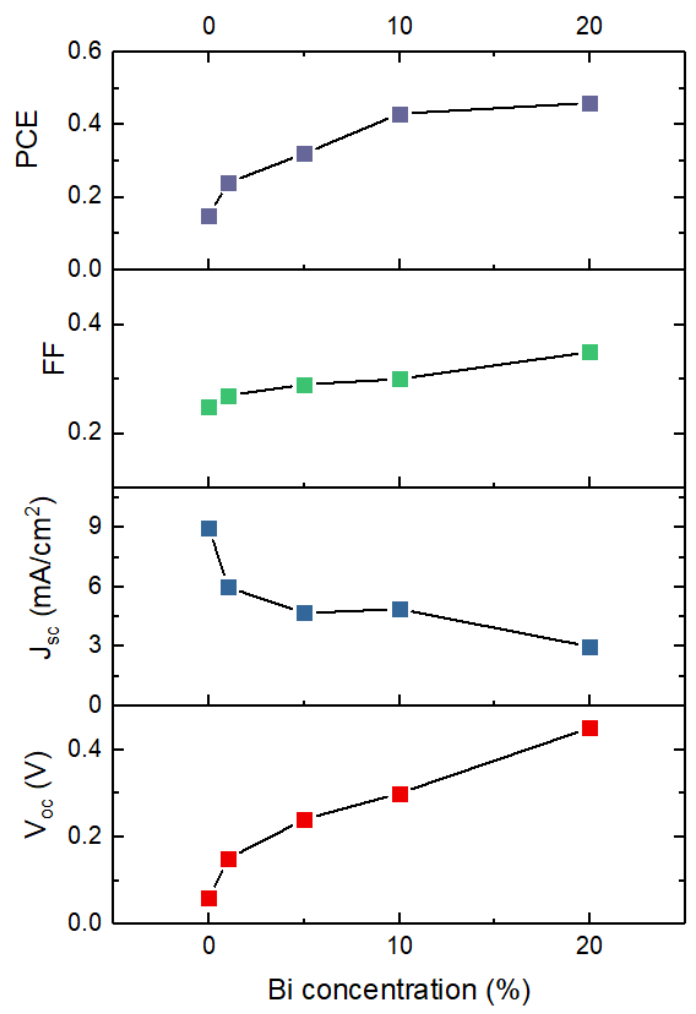

Figure S13. Impact of Bi doping on the solar cell device parameters.

The decrease of current density under dark condition, together with the enhanced photovoltaic effect support that the Bi incorporation reduces the tin vacancies and p-type self-doping in tin perovskite films. 


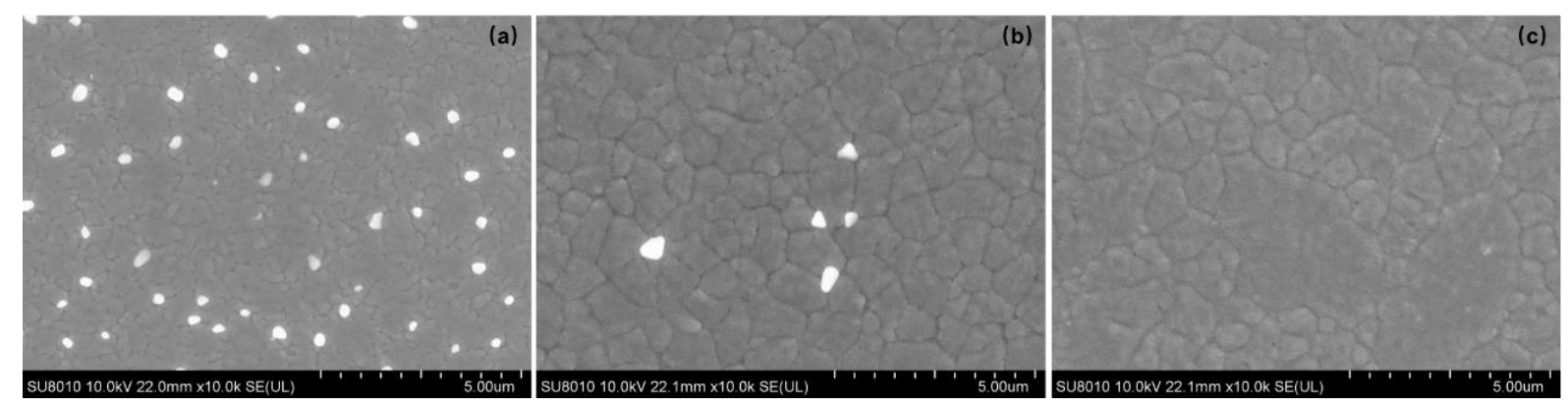

Figure S14. SEM images of undoped and Bi doped $\mathrm{CsSnI}_{3}$ films. (a) $0 \% \mathrm{BiI}_{3}$, (b) $1 \% \mathrm{BiI}_{3}$, and (c) $10 \%$ $\mathrm{BiI}_{3}$. 

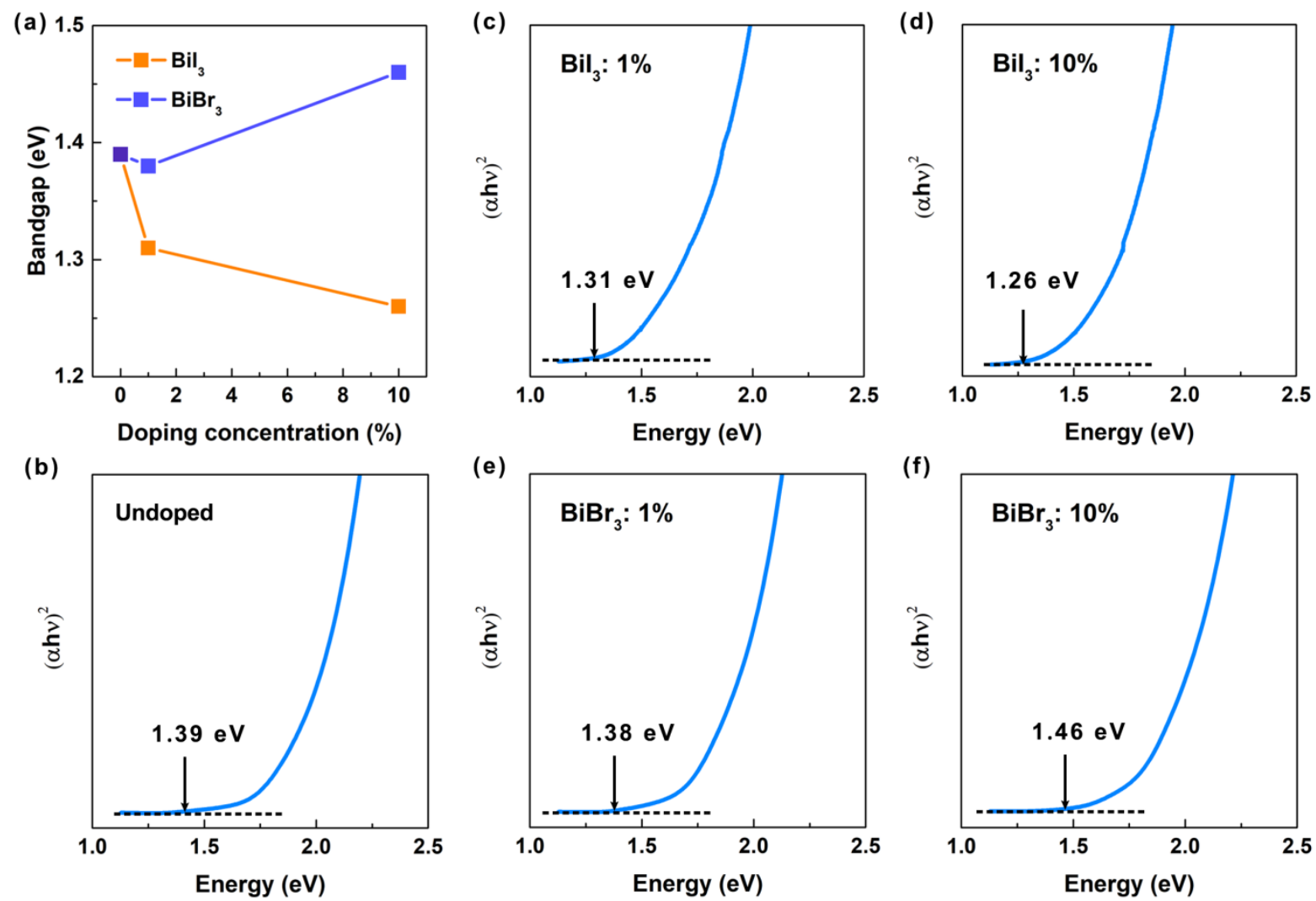

Figure S15. Impact of Bi doping on the optical absorption of $\gamma$-CsSnI $I_{3}$ films using different Bi precursors.

(a) $\mathrm{BiI}_{3}$, (b) $\mathrm{BiBr}_{3}$. The black arrow indicates the optical bandgap of $\gamma-\mathrm{CsSnI}_{3}$ films.

The optical absortion results support the succesful incoporation of $\mathrm{Bi}$ into $\mathrm{CsSnI}_{3}$ films. For undoped $\gamma$-CsSnI 3 films, the bandgap extracted from the absorption spectrum is $1.39 \mathrm{eV}$. After $\mathrm{BiI}_{3}$ doping, it gradually redshifts to $1.26 \mathrm{eV}\left(\mathrm{BiI}_{3} 10 \mathrm{~mol} \%\right.$ ), due to the substitution of $\mathrm{Sn}^{2+}$ by $\mathrm{Bi}^{3+}$. By contrast, the bandgap blueshifts to $1.48 \mathrm{eV}$ after $10 \mathrm{~mol} \% \mathrm{BiBr}_{3}$ doping, which can be ascribed to the partial substitution of $\mathrm{I}^{-}$by $\mathrm{Br}$. This is well-consistent with the more serious shift of XRD peak occurring to $\mathrm{BiBr}_{3}$ doped $\gamma-\mathrm{CsSnI}_{3}$ films. 
Table S1 The reported best $\mathrm{V}_{\text {oc }}$ for $\gamma$-CsSnI 3 based perovskite solar cells.

\begin{tabular}{|c|c|c|}
\hline & $\mathrm{V}_{\mathrm{oc}}(\mathrm{V})$ & Reference \\
\hline \multirow[t]{2}{*}{$\mathrm{CsSnI}_{3}$} & 0.24 & Adv. Mater. 2014, 26, 7122-7127 \\
\hline & & ACS Appl. Energy Mater.2018, 1, \\
\hline $\mathrm{CsSnI}_{3}$ & 0.34 & $4221-4226$ \\
\hline $\mathrm{CsSnI}_{3}$ & 0.45 & Nature Energy 2016, 1, 16178 \\
\hline $\mathrm{CsSnI}_{3}$ & 0.38 & ACS Energy Lett. 2017, 2, 897-903 \\
\hline \multirow[t]{2}{*}{$\mathrm{CsSnI}_{3}$} & 0.27 & Sol. RRL 2018, 2, 1700224 \\
\hline & & ACS Appl. Mater. Interfaces 2021, \\
\hline \multirow[t]{2}{*}{$\mathrm{CsSnI}_{3}$} & 0.46 & $13,1345-1352$ \\
\hline & & ACS Energy Lett 2021, 6, \\
\hline \multirow[t]{2}{*}{$\mathrm{CsSnI}_{3}$} & 0.64 & $1480-1489$ \\
\hline & & J. Am. Chem. Soc. 2021, 143, 11, \\
\hline $\mathrm{CsSnI}_{3}$ & 0.45 & $4319-4328$ \\
\hline $\mathrm{CsSnI}_{3}$ & 0.45 & This work \\
\hline
\end{tabular}




\section{References}

1 Shang, C., Liu, Z.-P. Stochastic Surface Walking Method for Structure Prediction and Pathway Searching. Journal of Chemical Theory and Computation 2013, 9, 1838-1845.

2 Shang, C., Zhang, X.-J., Liu, Z.-P. Stochastic surface walking method for crystal structure and phase transition pathway prediction. Physical Chemistry Chemical Physics 2014, 16, 17845-17856.

3 Huang, S.-D., Shang, C., Zhang, X.-J., Liu, Z.-P. Material discovery by combining stochastic surface walking global optimization with a neural network. Chemical Science 2017, 8, 6327-6337.

4 Zhu, S., Hu, Q., Mao, W. L., Mao, H., Sheng, H. Hydrogen-Bond Symmetrization Breakdown and Dehydrogenation Mechanism of $\mathrm{FeO}_{2} \mathrm{H}$ at High Pressure. Journal of the American Chemical Society 2017, 139, 12129-12132.

5 Zhang, X.-J., Liu, Z.-P. Variable-Cell Double-Ended Surface Walking Method for Fast Transition State Location of Solid Phase Transitions. Journal of Chemical Theory and Computation, 2015, 11, 4885-4894.

6 Kresse, G., Furthmüller, J. Efficient iterative schemes for ab initio total-energy calculations using a plane-wave basis set. Physical Review B 1996, 54, 11169-11186.

7 Blöchl, P. E. Projector augmented-wave method. Physical Review B 1994, 50, 17953-17979.

8 Perdew, J. P., Burke, K., Ernzerhof, M. Generalized Gradient Approximation Made Simple. Physical Review Letters 1996, 77, 3865-3868.

9. Heyd, J.; Scuseria, G.E.; Ernzerhof, M. Hybrid functionals based on a screened coulomb potential. Journal of Chemical Physics 2006, 124, 8207-8215.

10. Monkhorst, H.J.; Pack, J.D. Special points for Brillouin-zone integrations. Physical Review B 1976, 13, 5188-5192.

11. Yang, J.H.; Yin, W.J.; Park, J.S.; Ma, J.; Wei, S.H. Review on first-principles study of defect properties of CdTe as a solar cell absorber. Semiconductor Science and Technology 2016, 31, 083002.

12. Deng, H. X., Cao R. Y., Wei, S. H. First-principles study of defect control in thin-film solar cell materials. SCIENCE CHINA Physics, Mechanics \& Astronomy. 2021, 64, 237301. 